\title{
Effectiveness of Virtual Open Laboratory Teaching Assistant for Circuits Laboratories
}

\section{Mr. Firdous Saleheen, Temple University}

Firdous Saleheen received the B.Sc. degree in electrical and electronic engineering from Bangladesh University of Engineering and Technology (BUET), Dhaka, Bangladesh, in 2008, and the M.S. degree in electrical engineering from Temple University, Philadelphia, PA, USA in 2013. From 2008 to 2010, he was with Mango Teleservices Ltd., Dhaka, an international IP bandwidth provider of Bangladesh, as a Senior Engineer in the Research and Development Department. He is currently pursuing the Ph.D. degree in electrical engineering in ECE department of Temple University. His research interests include tactile sensation imaging, diffuse optical imaging, biomedical imaging systems development, machine learning, and statistical control theory.

\section{ZIcong Wang, Temple University}

Ph.D student in Temple University, Electrical and Computer engineering department.

\section{Mr. William Moser, Temple University}

William Moser is an M.S.E.E. student at Temple University's Control, Sensor, Network, and Perception (CSNAP) Laboratory.

\section{Vira Oleksyuk, Temple University}

Vira Oleksyuk received the B.S. degree in electrical and computer engineering with bio-electrical concentration from Temple University, Philadelphia, PA, USA, in 2013. She is a member of the Control, Sensor, Network, and Perception Laboratory, Temple University, where she is currently pursuing the Ph.D. degree in engineering, and a recipient of NSF Graduate Research Fellowship' 13. Her research interests are tactile imaging for breast cancer diagnostic, tissue spectroscopy, digital image processing, machine learning, and pattern recognition.

\section{Dr. Joseph Picone, Temple University}

Joseph Picone received his Ph.D. in Electrical Engineering in 1983 from the Illinois Institute of Technology. He is currently a Professor in the Department of Electrical and Computer Engineering at Temple University. His primary research interests are currently machine learning approaches to acoustic modeling in speech recognition. His research group is known for producing many innovative open source materials for signal processing including a public domain speech recognition system. He is a Senior Member of the IEEE and has been active in several professional societies related to human language technology. He has authored numerous papers on the subject and holds several patents in this field.

\section{Dr. Chang-Hee Won, Temple University}

Chang-Hee Won is an associate professor of electrical and computer engineering in the Department of Electrical and Computer Engineering and the director of Control, Sensor, Network, and Perception (CSNAP) Laboratory at Temple University. Previous to coming to academia, he worked at Electronics and Telecommunications Research Institute as a senior research engineer. Currently, he is actively guiding various research projects funded by National Science Foundation, Pennsylvania Department of Health, and Department of Defense. His research interests include stochastic optimal control theory, sensing systems, and virtual laboratory assistant. 


\title{
Effectiveness of a Virtual Open Laboratory Teaching Assistant for Circuits Laboratories
}

\author{
Firdous Saleheen, Zicong Wang, William Moser, Vira Oleksyuk, Joseph Picone, Chang-Hee Won \\ Department of Electrical and Computer Engineering
}

Temple University

\begin{abstract}
The Virtual Open Laboratory Teaching Assistant (VOLTA) has been developed as a personal assistant for students engaged in introductory circuits laboratories. VOLTA consists of prelaboratory instructions, topic explanation videos, equipment usage videos, circuit simulations, and actual laboratories. This web-based software allows students to perform circuits labs in a self-paced manner. Using VOLTA, students can explore elementary circuit topics in a learning on demand mode. It guides students through the process of building, verifying, and troubleshooting a circuit. VOLTA allows students to do circuits laboratories on demand. Using VOLTA, students can complete a laboratory without a human teaching assistant. Recent enhancements include a circuit comparator and a hardware circuit tracer. The circuit comparator verifies the simulated circuit, while the hardware circuit tracer provides troubleshooting instructions for the hardware circuit. The effectiveness of VOLTA was evaluated by comparing an experimental group of students to a control group of students. The experimental group was taught by VOLTA, while the control group was enrolled in a traditional version of the laboratory. An analysis of variance (ANOVA) test revealed a $p$-value of $<0.001$ at a confidence level of $95 \%$, which provides sufficient evidence that the students taught with VOLTA performed better than the control group. VOLTA is an effective teaching tool because it enhances student performance and reduces the workload for human teaching assistants.
\end{abstract}

\section{Introduction}

Engineering education traditionally utilizes a closed laboratory environment to provide students with a hands-on experience. Closed laboratories have limited efficiency because of space and time $^{1}$. In order to mitigate these issues, educators have been exploring alternatives such as open laboratories ${ }^{2,3}$ over the past few decades.

The open laboratory is an emerging pedagogical model in engineering education where the students have the flexibility of repeating and refining their experiments at any time ${ }^{4}$. Open laboratories reduce scheduling conflicts and ensure effective utilization of space and equipment ${ }^{5,6}$. Though an open laboratory can positively impact the educational experience, it is labor intensive as it requires continuous teaching assistant support for the students. In a traditional closed laboratory, teaching assistants typically play a significant role in a student's success. Not all the students are able to receive on-demand help during the course hours, because the number of teaching assistants is often limited. It is similarly not feasible to provide $7 / 24$ support. In order to address this problem, we developed a system called the Virtual Open 
Laboratory Teaching Assistant (VOLTA) that provides automated on-demand instructional support ${ }^{7}$.

Previously, Butz et al. developed a universal virtual laboratory ${ }^{8}$ to assist individuals who do not have adequate mobility of their upper bodies to perform laboratory experiments. The universal virtual laboratory had circuit element recognition and natural language recognition features ${ }^{9,10}$. VOLTA was based on these successful approaches. Equipped with pre-lab testing and instruction, engineering design exercises, short topic explanation videos, instrumentation instruction (including safety), and a corresponding post-lab test module, VOLTA is able to provide on-demand, smart assistance to students. A preliminary study on the effectiveness of VOLTA showed that the students participating in VOLTA performed slightly better compared to the control group ${ }^{7}$. In that experiment, the ANOVA (analysis of variance) test was run on the gain score of the students. The gain score is the difference between the post- and pre-test score. Students using VOLTA did well compared to traditional students with a $p$-value of 0.117 at $80 \%$ significance level. The average gains of the students using VOLTA are better than the traditionally taught students in five lab assignments out of eleven assignments. In two lab assignments, both groups performed similar. In rest of the lab assignments traditionally taught students performed better.

In Spring 2015, VOLTA was revamped by implementing an additional feature of "hardware help" and utilized more "questions and answers" collected from the prior evaluation conducted in Fall $2014^{7}$ ? This paper presents an analysis of these results. The analysis shows that the Spring 2015 version of VOLTA increased [...the learning...] in circuits laboratories significantly. An ANOVA test demonstrated that students using VOLTA performed better compared to the control group (students conducting the same experiments in a traditional laboratory setting) with $p<$ 0.001 .

The rest of the paper is organized as follows: Sections 2 provides an overview of VOLTA's design and implementation. Section 3 discusses the evaluation methods used to assess effectiveness. Results are presented in Section 4. A discussion of the effectiveness of VOLTA is presented in Section 5. Conclusions and future work are presented in Section 6.

\section{An Overview of VOLTA's Design and Implementation}

In this section, we briefly discuss the design and implementation of VOLTA. A detailed description can be found here ${ }^{7}$.

\section{Design}

VOLTA comprises four software modules and a database. The modules are (i) Instructor module, (ii) Student module, (iii) Help module, and (iv) Circuit comparator and tracer module.

i) Instructor module: The instructors and teaching assistants perform administration tasks through the Instructor module. It provides authorization access to VOLTA software and the courses. The instructors also use the module for populating the lab course materials. The instructors can check the progress of the students in a lab. 
ii) Student module: The Student module is the students' portal for interacting with VOLTA. It provides step-by-step guidelines for completing an experiment. It also provides assessments of subject materials.

iii) Help module: The Help module provides students with virtual teaching assistance. Students can request assistance from this module at any point during a session. This module helps students with definitions of circuit terminology, questions and answers about basic electric and electronic circuit concepts related to the lab. There are also safety video tutorials, basic instrument use video tutorials, a forum for discussing problems, and video tutorials explaining how to use the system. The instructor can preload necessary materials in the Help module using the Instructor module.

iv) Circuit Comparator and Tracer module: This module verifies simulated circuits by comparing a student's netlist to a reference netlist. This module also provides troubleshooting steps for hardware implementations.

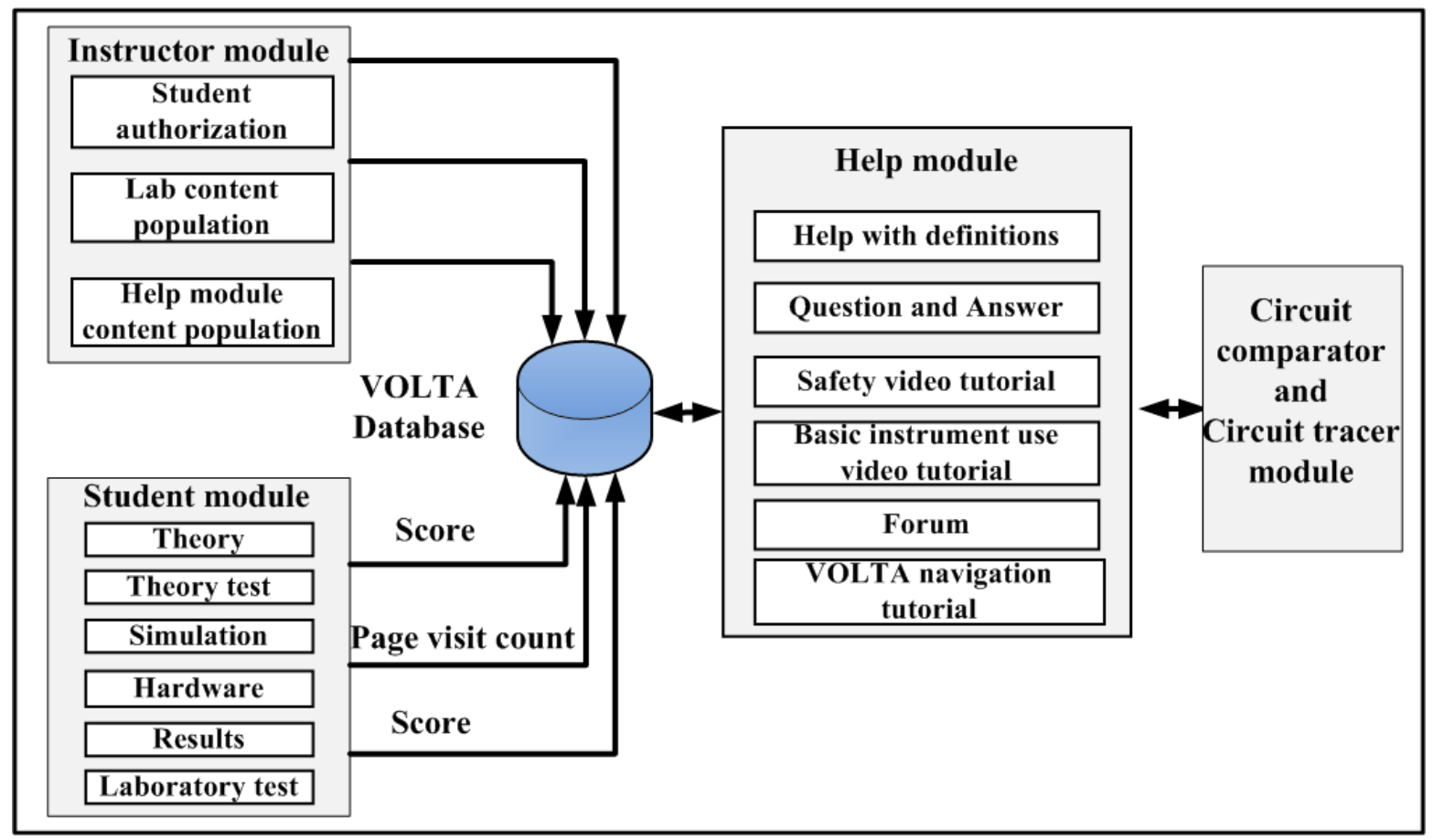

Figure 1: VOLTA modules

\section{Implementation}

VOLTA is implemented using Python (version 2.7.1) and Django (version 1.6.5). Django is a high-level Python web framework for rapid and scalable web development ${ }^{11}$. This framework consists of model, view, and template layers. It generates a dynamic webpage for each user. VOLTA provides a user-friendly administration panel which gives access in VOLTA database. The lab course materials can be loaded into VOLTA in two ways: via python scripts and via the administration panel. Before making the website live, it is more convenient to load the content using scripts. After making the website live, the administration panel is used for maintaining and updating content. In this way, one can easily avoid overwriting the current user information. 


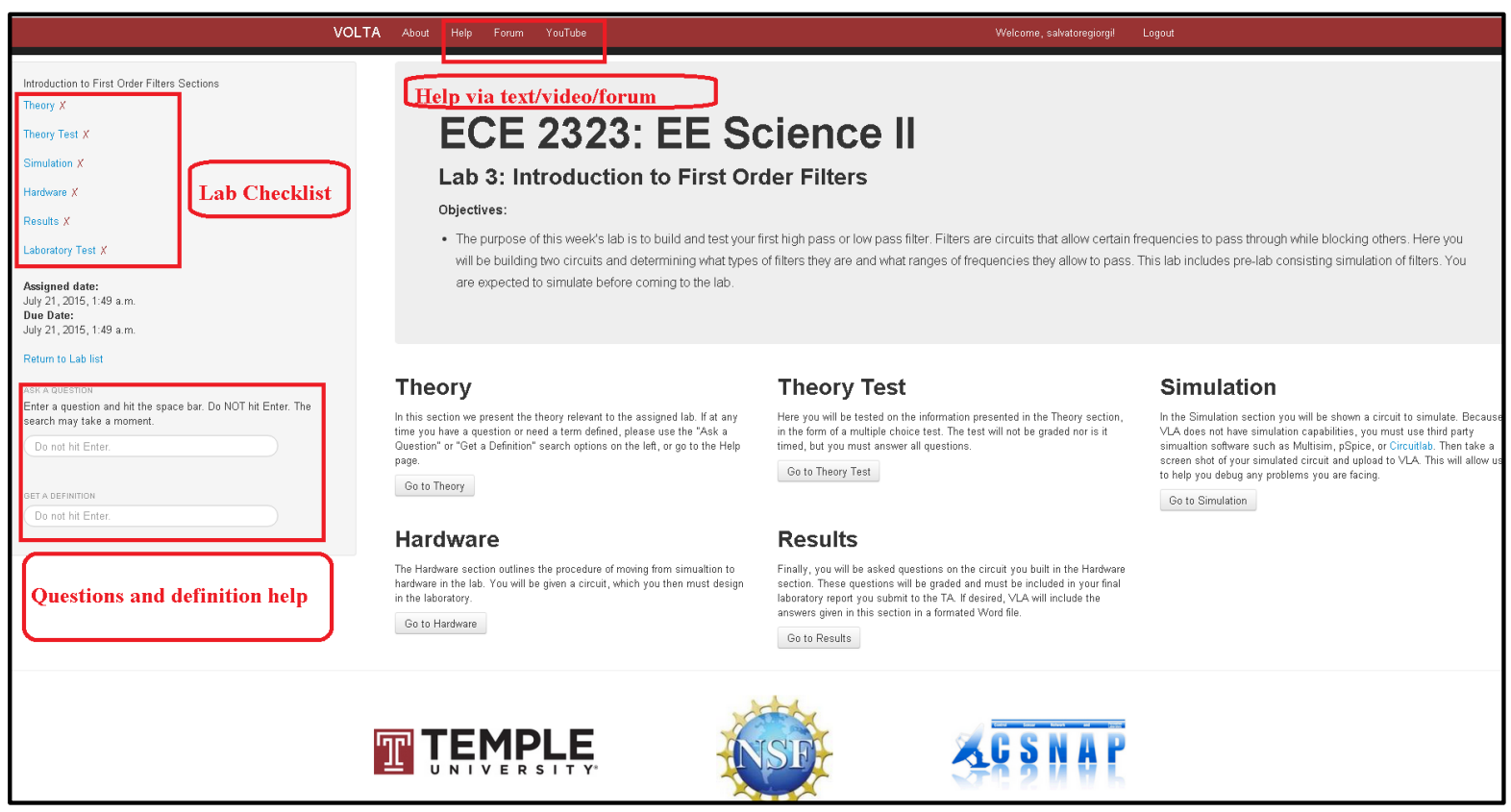

Figure 2: VOLTA user interface

VOLTA's user interface guides students through an experiment step-by-step as shown in Fig. 2. The students take part in pre-tests and post-tests that consist of multiple choice questions. The same set of questions are asked in pre- and post-tests. In post-tests, the multiple choice options are not in the same order as in pre-tests. After the pre-test, the students are directed to the simulation section. In this section, the students are instructed to simulate their circuits before constructing a hardware implementation.

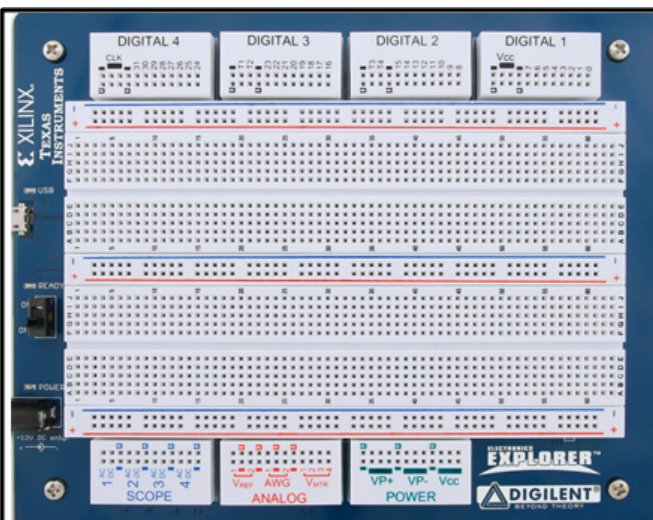

Electronics Explorer Board

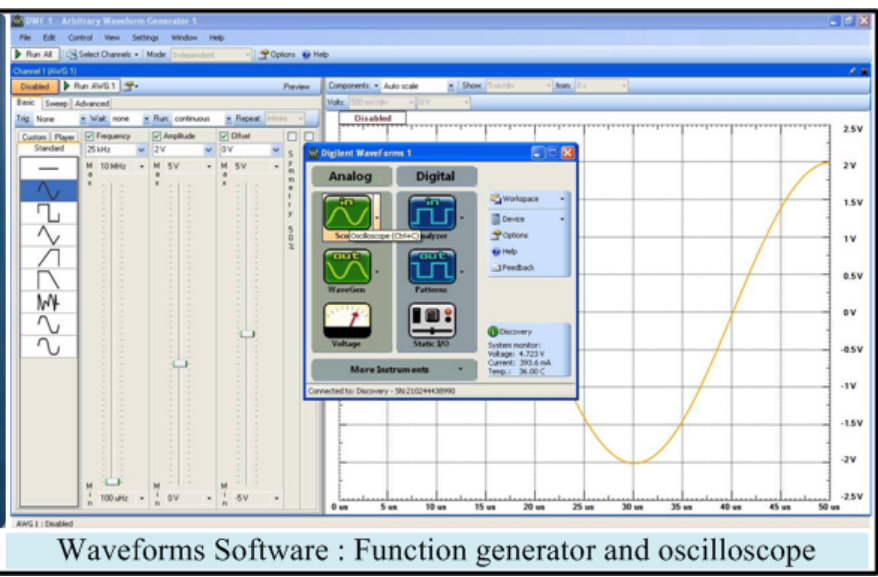

Figure 3: Electronics Explorer board and Waveforms software interface

The hardware section provides instructions for building a circuit on a breadboard. The student uses the Electronics Explorer (EE) board (Digilent Inc., Pullman, Washington, USA) for the hardware implementation ${ }^{12}$. The EE board is built around a solderless breadboard, which also includes oscilloscopes, waveform generators, power supplies, voltmeters, reference voltage generators, and thirty-two digital signals that can be configured as a logic analyzer, pattern generator, or any one of several static digital I/O devices. All of these instruments can be 
connected to circuits built on solderless breadboards using simple jumper wires. For data acquisition and analysis, PC based software named "WaveForms" is used. A high-speed USB 2.0 connection ensures near real time data acquisition.

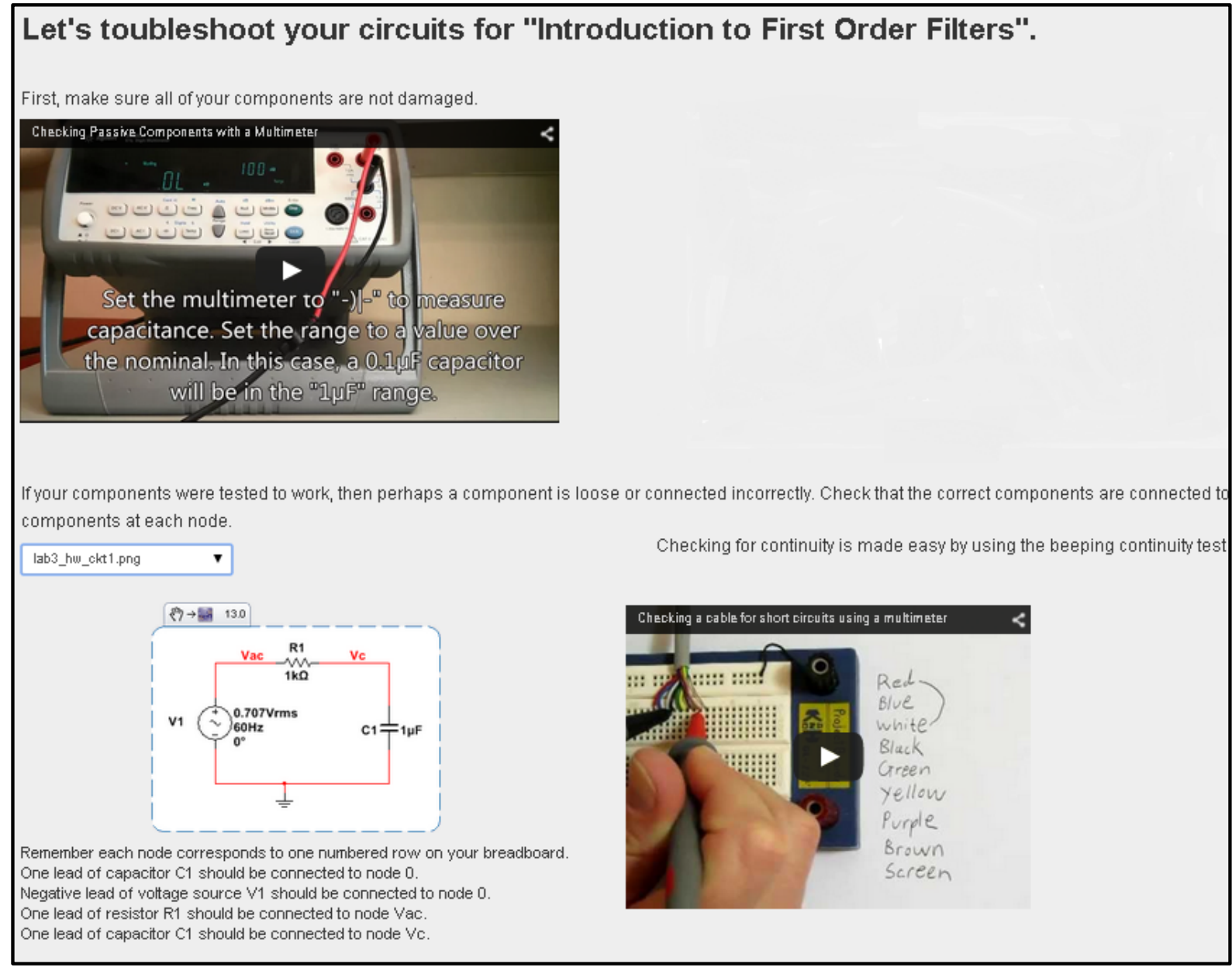

Figure 4: Circuit Tracer

In the hardware section, VOLTA provides assistance for tracing circuit connections. Fig. 4 shows the circuit tracer page for an experiment. The troubleshooting guide starts with the component integrity test. A video instruction is provided to check whether the components are damaged. Secondly, another video instruction shows how to do the continuity test to check whether there are any loose connections. A brief description of circuit node and component connectivity is given for the desired circuit. This description is generated from a previouslyloaded Multsim netlist of the circuit.

The Results section contains a guideline for the contents of report. At the end of the lab, the students take a laboratory-test which is a post-test in the form of multiple-choice based on the lab. 


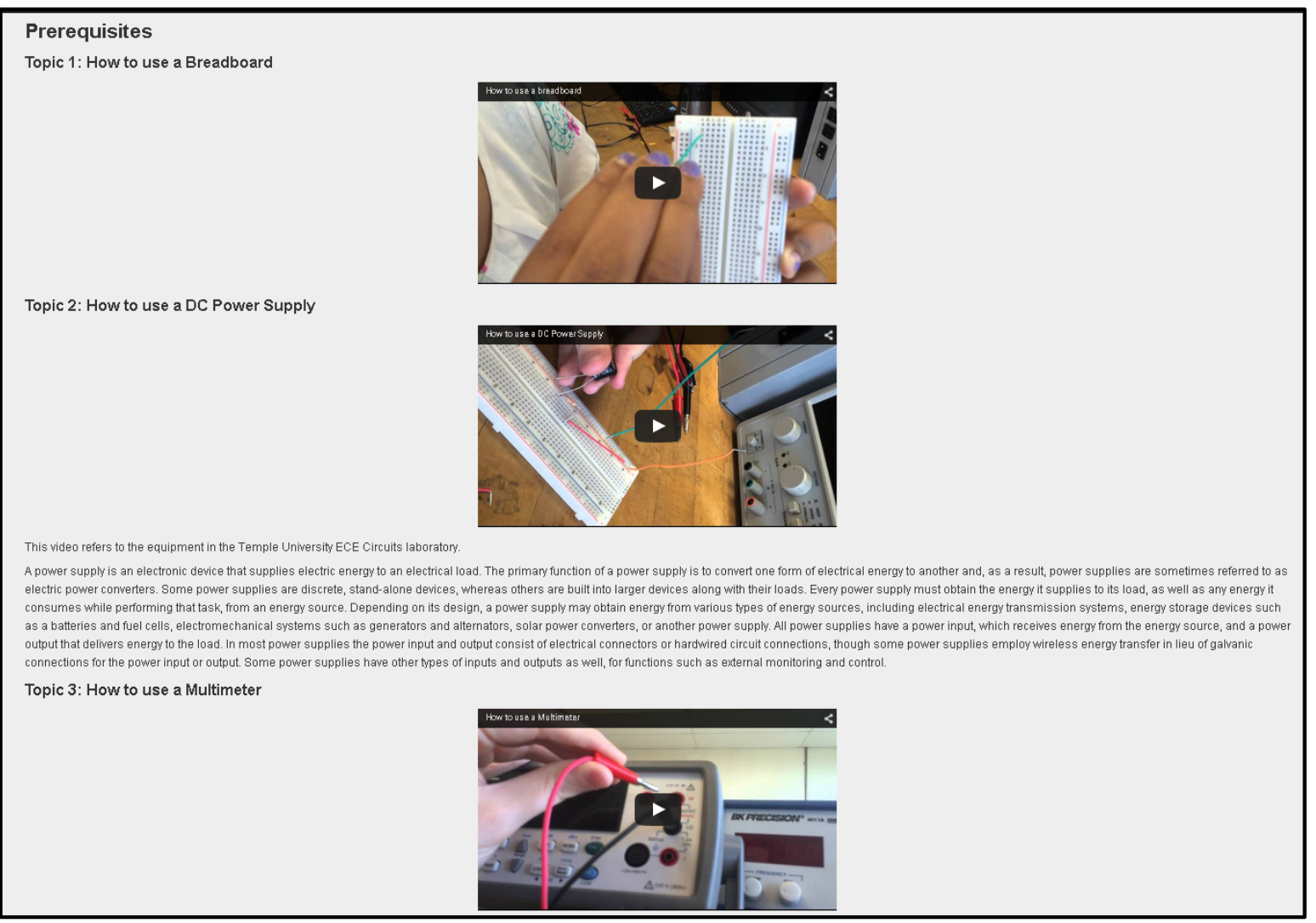

Figure 5: Video tutorials in VOLTA help page

The students can look for the questions, answers, and definitions in the help page. This page gives a summary list of the most viewed questions and definitions. Also, there are video tutorials for performing basic circuit laboratory work. Fig. 5 shows the prerequisite page with video instructions for doing lab-work.

\section{Evaluation of VOLTA}

VOLTA was evaluated from two perspectives: usability and effectiveness. Usability evaluation data provides knowledge about a program's functional effectiveness, efficiency, ease of learning, ease of use, motivational influence, and quality assurance. The effectiveness study of VOLTA provides insights about its usefulness compared to traditionally taught classes. IRB approval (\#22447) was obtained from Temple University.

\section{Usability}

A Likert scale was used for the usability evaluation, which is the most widely used technique to measure attitude ${ }^{13}$. It evaluates the attitude toward a topic by presenting a set of statements about the topic to the respondents. The respondents are asked to indicate whether they strongly agree, agree, disagree, strongly disagree, or hold no opinion about the statements. Their responses are assigned numeric values, e.g. strongly disagree $=1$, disagree $=2$, no opinion $=3$, agree $=4$ and 
strongly agree $=5$. The mean attitude score can then be calculated by averaging their response scores according to the Likert scale. A mean score below 3 implies a negative attitude and above 3 implies a positive attitude.

The survey includes 20 questions regarding the usability of VOLTA software. The 20 questions covered seven broad categories:

A. Did students think the VOLTA is useful for their learning? (Learning environment)

B. Did students find the software motivating? (Motivational value)

C. Did students find the VOLTA easy to use? (Ease of use)

D. Did students perceive the usefulness of various features of the VOLTA? (Perception of usefulness)

E. Did students "buy into" the virtual laboratory environment? (Authenticity of virtual learning)

F. What was the perceived quality of the VOLTA? (Quality assurance)

G. What additional features or learning situation the students would like to see? (Expectations)

\section{Effectiveness}

The study included students enrolled in a laboratory course on circuits. The lab had two sections. The students were randomly assigned to each section. The sections were randomly chosen as experimental and control groups. The students in the experimental group received a curriculum in which the instructor integrated VOLTA. The students in the control group received the traditional course curriculum. The effectiveness of VOLTA is assessed using gain score analysis of pre-test/post-test design ${ }^{14}$. Eleven lab assignments were developed for VOLTA. With each lab assignment, there was one pre- and post-test.

\section{Experimental Results}

During Spring 2015, the assessment data was collected from twenty-eight students using our student survey infrastructure. These students were enrolled in an introductory AC circuit lab "Electrical Engineering Science II". The experimental group consisted of 18 students, while the control group consisted of 10 students. The experimental and control groups were taught on different days and times of the week.

\section{Usability Results}

Seven out of 10 students completed the survey questionnaire. Fig. 6(a) shows category-wise mean attitude score. In all seven categories, the students showed a positive attitude. In category A, five Likert-type questions were provided to understand how successful VOLTA was as a learning environment. At least $57 \%$ of the responses agreed that they viewed VOLTA as a useful learning tool. In category $\mathrm{B}$, two questions were provided to assess the motivational value of VOLTA. At least $43 \%$ of the students agreed that they enjoyed using VOLTA. In category C, three questions probed the ease of use of VOLTA. 99\% of the students found VOLTA easy to use and easy to navigate. The students also felt that the use of VOLTA was intuitive $(85 \%)$. In 
category D, four questions were asked regarding to what degree VOLTAs features were helpful. $71 \%$ of the students thought the VOLTA Youtube channel was useful. The students indicated that the instructional videos were the most helpful features of VOLTA.

In category E, two questions were used to assess the authenticity of virtual learning. 57\% of the students felt the labs seemed like a real lab. In category F, two questions were used to assess the quality of the videos and VOLTA overall. In both cases, $85 \%$ of the students agreed the videos were of good quality and VOLTA was trouble-free. In category G, two questions probed expectations. $56 \%$ of the students expected a feature to verify their simulation results in VOLTA. Fig. 6 (b) shows the mean attitude scores of seven individuals. All of them except one showed positive attitude towards VOLTA.

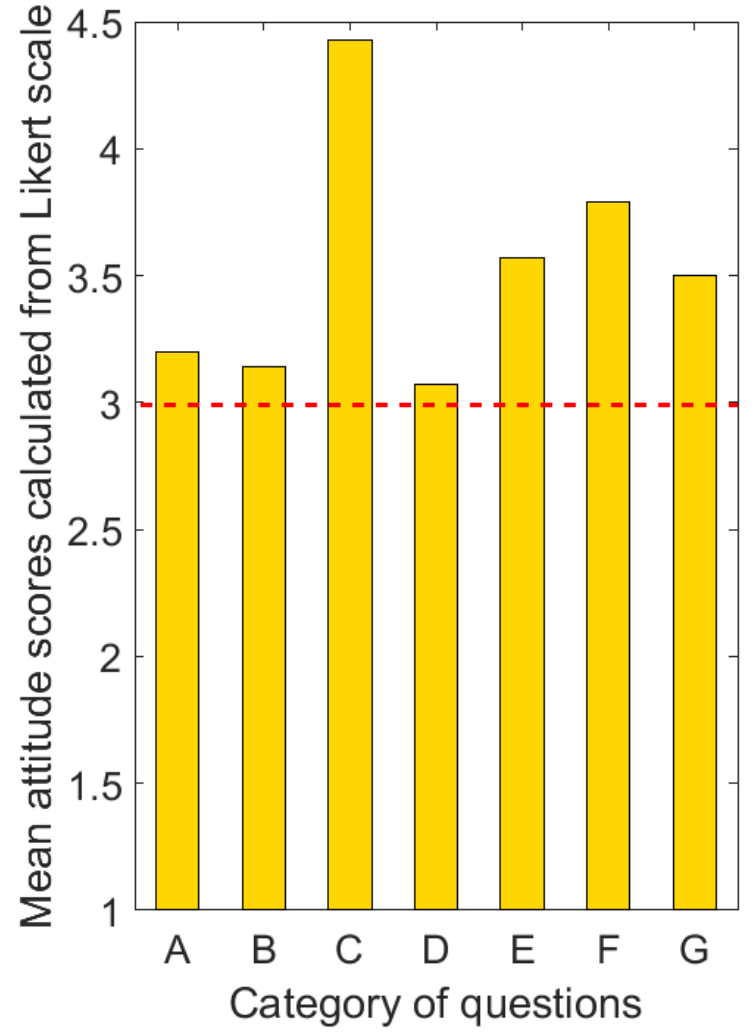

(a)

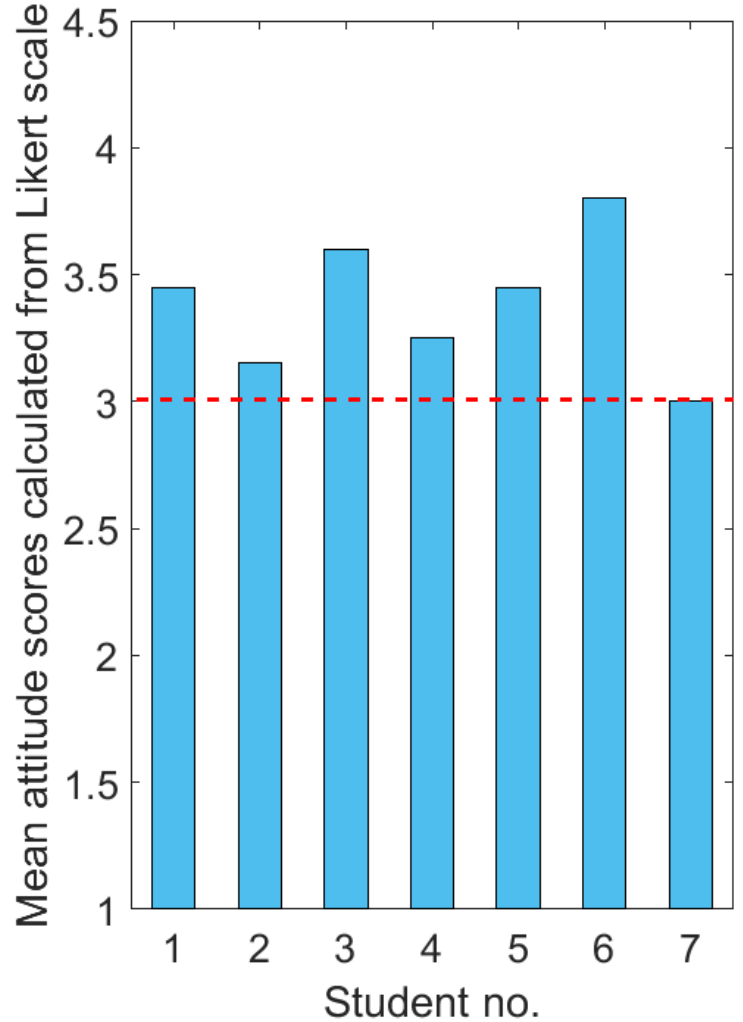

(b)

Figure 6: (a) Mean attitude scores for each category of questions. (b) Mean attitude scores of students towards VOLTA. The negative attitude region lies below the dotted line. 


\section{Effectiveness Results}

A total of eleven lab assignments were performed in eleven weeks. For each of the lab assignments, there were pre-lab and post-lab tests. The pre-lab test comprised five questions in multiple choice formats. Each question carried 20 points. The post-lab test comprised the same five questions with the multiple choice answers in a randomized order. The test solutions were not revealed until the post-lab tests were completed. Also, the VOLTA help module does not provide any material directly related to the tests. The same instructor taught both sections. Table 1 and 2 show the pre-test post-test scores of the control and experimental group of students, respectively. The average gain is the difference between the mean post- and pre-test score.

Table 1: Control Group's (n=10) Pre-test and Post test Score

\begin{tabular}{|c|c|c|c|c|}
\hline \multirow{2}{*}{$\begin{array}{c}\text { Lab } \\
\text { Assignment } \\
\text { no. }\end{array}$} & \multicolumn{2}{|r|}{ Pre-test } & \multicolumn{2}{|r|}{ Post-test } \\
\hline & Mean & Standard Deviation & Mean & Standard Deviation \\
\hline 1 & 61.11 & 19.40 & 68.89 & 15.23 \\
\hline 2 & 48.89 & 23.31 & 54.44 & 8.96 \\
\hline 3 & 67.06 & 13.62 & 68.24 & 13.82 \\
\hline 4 & 74.44 & 26.50 & 83.33 & 30.73 \\
\hline 5 & 85.56 & 17.39 & 84.44 & 15.71 \\
\hline 6 & 77.78 & 22.00 & 77.78 & 20.96 \\
\hline 7 & 54.44 & 23.86 & 47.78 & 23.23 \\
\hline 8 & 76.67 & 30.00 & 81.11 & 27.06 \\
\hline 9 & 85.56 & 17.39 & 83.33 & 17.95 \\
\hline 10 & 54.44 & 28.91 & 54.44 & 29.67 \\
\hline 11 & 47.78 & 20.15 & 48.89 & 20.25 \\
\hline
\end{tabular}

Table 2: Experimental Group's $(\mathrm{n}=18)$ Pre-test and Post test Score

\begin{tabular}{|c|c|c|c|c|}
\hline \multirow{2}{*}{$\begin{array}{c}\text { Lab } \\
\text { Assignment } \\
\text { no. }\end{array}$} & \multicolumn{2}{|r|}{ Pre-test } & \multicolumn{2}{|r|}{ Post-test } \\
\hline & Mean & Standard Deviation & Mean & Standard Deviation \\
\hline 1 & 68.00 & 16.00 & 76.00 & 8.00 \\
\hline 2 & 44.00 & 12.00 & 56.00 & 12.00 \\
\hline 3 & 76.00 & 12.00 & 88.00 & 9.80 \\
\hline 4 & 54.00 & 20.10 & 66.00 & 26.91 \\
\hline 5 & 84.00 & 24.98 & 90.00 & 18.44 \\
\hline 6 & 76.00 & 26.53 & 90.00 & 18.44 \\
\hline 7 & 62.00 & 10.77 & 68.00 & 9.80 \\
\hline 8 & 70.00 & 28.64 & 84.00 & 14.97 \\
\hline 9 & 70.00 & 18.44 & 96.00 & 8.00 \\
\hline 10 & 56.00 & 24.98 & 74.00 & 20.10 \\
\hline 11 & 74.00 & 15.62 & 76.00 & 14.97 \\
\hline
\end{tabular}



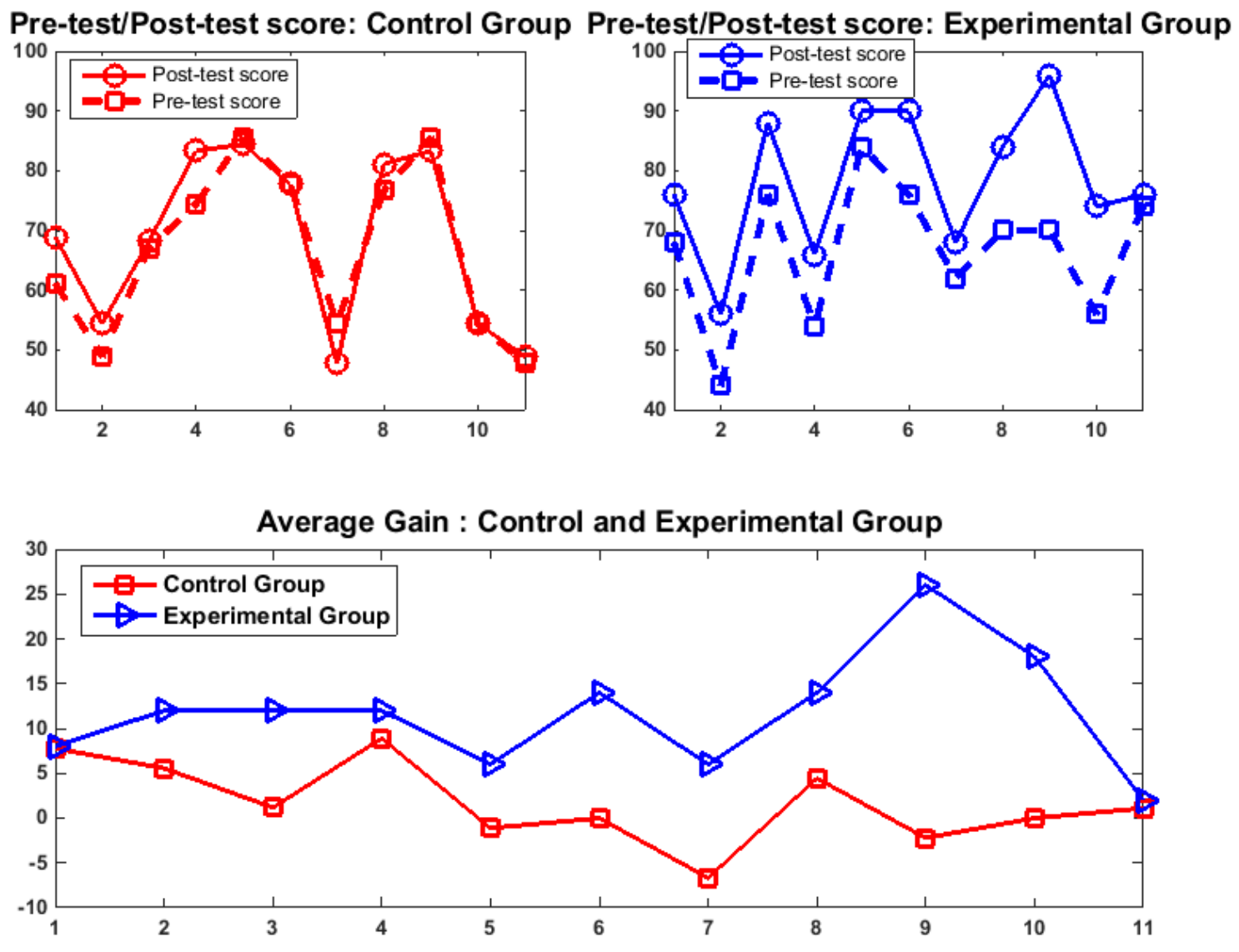

Figure 8: Pre-test and Post-test score

The same instructor taught both sections. Table I shows the mean and standard deviation of pretest and post-test scores of the control and experimental group of students for each lab assignment. The average gain is the difference between the mean post- and pre-test score. Fig. 8 shows plots of pre-test and post-test score versus lab assignment index and average gain versus lab assignment index. From the figure, it can be seen that students taught by VOLTA performed significantly better in lab assignments $2,3,4,5,6,7,8,9$, 10, whereas traditionally-taught students scored better in lab assignments 1 and 11 .

A two-group pre-test/post-test design approach was used to evaluate the effectiveness of VOLTA. The major question guiding the evaluation of VOLTA's effectiveness on learning was "Did the students who used VOLTA (experimental group) learn more (e.g. score higher on gain measures) than their counterparts in the control group?" Gain score analysis was used to analyze data from the two-group pre-test/post-test research design. The gain score was again defined as the difference between the post-test and pre-test scores. The null hypothesis is that there is no difference among the mean gains of the experimental and control groups for eleven assignments. The ANOVA ${ }^{15}$ test was performed on the gain scores of 28 students in 11 lab assignments. The ANOVA test was performed using $\mathrm{R}$ (version 3.1.2). Table 3 shows the ANOVA result. A pvalue $<0.001$ indicates that the null hypothesis can be rejected. 
Table 3: ANOVA results to test the effect of VOLTA on students' gain score

\begin{tabular}{lrrrrr}
\hline $\begin{array}{l}\text { Source of } \\
\text { Variation }\end{array}$ & $\begin{array}{c}\text { Degrees } \\
\text { of } \\
\text { freedom }\end{array}$ & $\begin{array}{c}\text { Sum of } \\
\text { Squares }\end{array}$ & $\begin{array}{c}\text { Mean } \\
\text { Square }\end{array}$ & F ratio & $\boldsymbol{p}$-value \\
\hline Lab assignment no. & 10 & 3,782 & 378.2 & & \\
Group & 1 & 7,215 & 7215 & 27.15 & $<0.001$ \\
Residual & 296 & 78,671 & 266 & & \\
Total & 307 & 89,568 & & & \\
\hline
\end{tabular}

\section{Discussions}

The results from this study were encouraging and showed the benefits of VOLTA. The effectiveness assessment showed VOLTA students performed better than those of traditional lab students in eleven pairs of similar tests. VOLTA students were taught in a similar way as the traditional lab students, except without any handouts. Outside the lab, VOLTA students obtained help from VOLTA any time and from TAs during office hours. The traditional lab students got help from TAs only. VOLTA students received a greater amount of help compared to the traditional students, which was reflected in the effectiveness analysis. The Spring 2015 version of VOLTA achieved $p<0.001$, which was much better than the Fall 2014 version.

The Spring 2015 version of VOLTA had one new feature "Hardware Help", which provides a hardware assembly instructions based on Multisim netlist. Also, the Help module of Spring 2015 version of VOLTA had more questions and answers included. In Fall 2014, VOLTA was used for the first time. The feedback in Fall 2014 enriched the Spring 2015 VOLTA.

The survey and in-class discussion showed that the students require some time to use VOLTA efficiently, but the period was fairly short. After the fourth week, VOLTA students seemed to learn more, which was reflected in their post-test results from Lab 5. The students also felt that a verification of their simulation and hardware circuits would help them to a great extent. That justified the development of a circuit recognizer for VOLTA.

Finally, the implementation of VOLTA initially required a substantial amount of time. Creating lab contents and videos required a significant time investment by the instructor and TA. Additional time was required to identify the questions the students might ask during the lab. Once the course material was developed, however, TAs were not required to spend as much preparing for each lab compared to the traditional lab. Most of the course materials are reusable.

\section{Conclusions}

VOLTA is an intelligent tutoring system that provides students with an open laboratory environment equipped with virtual teaching assistance. VOLTA provides a self-paced environment, on-demand help, and increasing levels of engagement. The students gave positive feedback on various VOLTA content, such as instructional videos, safety videos, and short topic explanations. The students taught by traditional lectures and VOLTA were compared using the 
pre- and post-test performance. The ANOVA test showed $p<0.001$ indicating VOLTA had a significant effect on students' performance. With the circuit comparator and circuit tracer features, VOLTA reduces the students' dependency on human teaching assistants. On the other hand, VOLTA decreases teaching assistants' workloads by providing reusable course materials. Therefore, VOLTA can be instrumental for teaching circuits laboratory effectively.

\section{Acknowledgement}

This work was supported by a grant from the National Science Foundation grant number DUE 1245277. This work was partially funded by the Temple University College of Engineering Summer Undergraduate Research Program. We thank Dr. Richard Heiberger, Professor Emeritus of the Statistics Department at Temple University for his contribution in data analysis. We are grateful to Dr. Brian Butz, Professor Emeritus of the ECE Department for his insightful remarks on the development of the framework. 


\section{Bibliography}

1. C. Knight and S. DeWeerth, "A shared remote testing environment for engineering education," in Frontiers in Education Conference, 1996. FIE '96. 26th Annual Conference., Proceedings of, vol. 3, Salt Lake City, UT, USA, Nov 1996, pp. 1003-1006.

2. Educating the Engineer of 2020: Adapting Engineering Education to the New Century. Washington, DC: The National Academies Press, 2005.

3. R. Adams, D. Evangelou, L. English, A. D. De Figueiredo, N. Mousoulides, A. L. Pawley, C. Schiefellite, R. Stevens, M. Svinicki, J. M. Trenor, and D. M. Wilson, "Multiple perspectives on engaging future engineers," Journal of Engineering Education, vol. 100, no. 1, pp. 48-88, 2011.

4. J. Palais and C. Javurek, "The arizona state university electrical engineering undergraduate open laboratory," Education, IEEE Transactions on, vol. 39, no. 2, pp. 257-264, May 1996.

5. J. A. Oswald and M. E. Sloan, "An economical self-supervised individually operated open electronics laboratory," Education, IEEE Transactions on, vol. 14, no. 3, pp. 90-94, Aug 1971.

6. W. Kuhn, D. R. Hummels, and S. Dyer, "A senior-level rf design course combining traditional lectures with an open laboratory format," in Frontiers in Education Conference, 2000. FIE 2000. 30th Annual, vol. 1, 2000, pp. $\mathrm{T} 1 \mathrm{D} / 19-\mathrm{T} 1 \mathrm{D} / 23$.

7. F. Saleheen, S. Giorgi, Z. Smith, J. Picone, and C.-H. Won, "Design and evaluation of a web-based virtual open laboratory teaching assistant (volta) for circuits laboratory," in Proc. ASEE Annual Conference, Seattle, WA, USA, June 2015, pp. 1-16, paper ID 11469.

8. B. P. Butz, M. Duarte, and S. Miller, "An intelligent tutoring system for circuit analysis," Education, IEEE Transactions on, vol. 49, no. 2, pp. 216-223, May 2006.

9. M. Duarte, B. P. Butz, S. Miller, and A. Mahalingam, "An intelligent universal virtual laboratory (uvl)," Education, IEEE Transactions on, vol. 51, no. 1, pp. 2-9, Feb 2008.

10. K. Krishnasamy, B. P. Butz, and M. Duarte, "A rule-based semiautomated approach to building natural language question answering (nlqa) systems," Jacksonville, FL, USA, Jan. 2004.

11. https://www.djangoproject.com/

12. https://www.digilentinc.com/data/Products/EEBOARD/Digilent-EEboard-np-07.pdf

13. D. Ary, L. Jacobs, A. Razavieh, and C. Sorensen, Introduction to Research in Education, ser. SOE Curriculum Lab. Cengage Learning, 2009.

14. Dimiter M. Dimitrov and Phillip D. Rumrill, Jr., "Pretest-posttest designs and measurement of change", IOS press, Work 20 (2003) 159-165.

15. Montgomery, D.C., "Design and Analysis of Experiments", John Wiley \& Sons, Incorporated, 2012. 